\title{
Article \\ Effects of Hydroxychloroquine on Retinal Vessel Density in Patients with Rheumatoid Arthritis over One-Year Follow-Up: A Pilot Study
}

\author{
Pierluigi Iacono ${ }^{1, *++}$ (D) , Stefano Da Pozzo ${ }^{1}$, Alberto Bedendo ${ }^{2}$, Monica Varano ${ }^{3}$ and Mariacristina Parravano ${ }^{3}$ \\ 1 TS Retina, 34100 Trieste, Italy; stefanodapozzo@yahoo.it \\ 2 ASL 5, 33170 Pordenone, Italy; albertobedendo@virgilio.it \\ 3 IRCCS—Fondazione Bietti, 00198 Rome, Italy; m.varano@mclink.it (M.V.); mcparravano@gmail.com (M.P.) \\ * Correspondence: pierluigi.iacono@libero.it; Tel./Fax: +39-0481-533578 \\ † Current address: Via N. Sauro 8, 34170 Gorizia, Italy.
}

check for

updates

Citation: Iacono, P.; Da Pozzo, S.; Bedendo, A.; Varano, M.; Parravano, M. Effects of Hydroxychloroquine on Retinal Vessel Density in Patients with Rheumatoid Arthritis over One-Year Follow-Up: A Pilot Study. Appl. Sci. 2021, 11, 9837. https:// doi.org/10.3390/app11219837

Academic Editor: Taeyoon Son

Received: 10 September 2021

Accepted: 19 October 2021

Published: 21 October 2021

Publisher's Note: MDPI stays neutra with regard to jurisdictional claims in published maps and institutional affiliations.

Copyright: (c) 2021 by the authors. Licensee MDPI, Basel, Switzerland. This article is an open access article distributed under the terms and conditions of the Creative Commons Attribution (CC BY) license (https:// creativecommons.org/licenses/by/ $4.0 /)$.

\begin{abstract}
Background: To employ optical coherence tomography angiography (OCTA) to evaluate the effects of hydroxychloroquine (HCQ) on the superficial capillary plexus (SCP) and deep retinal capillary plexus (DCP) in patients affected by rheumatoid arthritis (RA). Methods: Patients with recent diagnosis of "definite RA", based on 2010 Rheumatoid Arthritis Classification Criteria, were included in a prospective, observational imaging study carried out by the G.B. Bietti Foundation between March 2019 and January 2020. Vessel density (VD) of SCP and DCP, central foveal thickness (CFT) and foveal avascular zone (FAZ) values were collected by OCTA. The primary outcome measure was the VD alteration of SCP and DCP in RA-patients after one year of HCQ treatment. Results: OCTA data analysis showed no statistically significant reduction in the mean VD of SCP and DCP, including the mean global area, central subfield, inner ring and temporal, superior, nasal, and inferior sectors, as well as in the mean CFT and FAZ areas. Conclusions: OCTA demonstrated no early change in the VD in the SCP and DCP, in RA-patients after one year of HCQ treatment. A longer monitoring period would more precisely establish the treatment's effect on the VD and its correlation with HCQ toxicity.
\end{abstract}

Keywords: deep retinal capillary plexus; optical coherence tomography angiography; rheumatoid arthritis; superficial retinal capillary plexus; hydroxychloroquine

\section{Introduction}

Chloroquine and hydroxychloroquine (HCQ) are frequently used as first-line treatment in patients with autoimmune diseases, including rheumatoid arthritis (RA), systemic lupus erythematosus (SLE), and Sjogren's syndrome [1-5]. The choice often falls on these drugs because of the rarity of the toxic effects associated with them-mainly involving dyspepsia, nausea, vomiting, abdominal pain, rush and nightmares [6]. Particular attention is focused on retinal toxicity, which seems to be positively related to the daily dose, the cumulative dose and the duration of the treatment [7]. The mechanisms by which chloroquine/HCQ toxicity are expressed have not yet been fully determined.

These drugs' high binding affinity with the melanin present in the retinal pigment epithelium (RPE), leading to RPE and photoreceptor cell damage, seems to be the most favored hypothesis [8-10]. A more recent opinion suggests the retinal microcirculation might play a role in the evolution of retinal damage secondary to the administration of HCQ [11-16]. Briefly, HCQ is thought to induce a remodeling of the retinal microcirculation by reducing vessel density (VD), retinal capillary plexuses and choriocapillaris, as revealed by optical coherence tomography angiography (OCTA), eventually leading to a reduced blood flow and hypoxic-ischemic damage. 
However, this hypothesis does not take into account possible anomalies in the retinal microcirculation secondary to autoimmune diseases. Primary changes in the circulation of large vessels and peripheral microvessels in SLE and RA patients have been described [17-23]. Alterations of the retinal macrocirculation in association with RA have also been previously identified [24]. In a recent study employing OCTA, we detected a reduced VD in the superficial retinal capillary plexuses in naïve patients diagnosed with early-stage definite RA, compared with a healthy control group [25]. Primary anomalies of the retinal microvascular system might therefore be characteristic of this rheumatic disease's pathological vascular mechanism, rather than being secondary to antimalarial drug toxicity.

The aim of the current study is to evaluate the effects of HCQ on the VD of capillary retinal plexuses in treatment-naive patients with definite RA, after one year of follow-up.

\section{Materials and Methods}

The current study, examining treatment-naïve patients affected by RA, was prospectively carried out at the Department of Ophthalmology of the G. B Bietti Foundation in Rome. The study was conducted in accordance with the Declaration of Helsinki, and the protocol was approved by the Ethics Committee of Fondazione Bietti- IFO (Project identification code: N.114/21/FB). Each patient was carefully informed about the purpose of the research and provided signed consent to all procedures and data analysis.

The study adopted inclusion and exclusion criteria as follows: Recent diagnosis of "definite RA", based on 2010 Rheumatoid Arthritis Classification Criteria. Patients were included with a score greater than 6 with regard to the following four main criteria: joint involvement, serology, acute-phase response measure, and symptom duration [26]; no previous or current treatment related to RA; no concomitant systemic disorders, including diabetes, cardiovascular diseases, and hypertension; no previous or concomitant ocular disease able to confound the appropriate examination, including media opacity and pathologic myopia, especially any type of retinal disorder; and no history of ocular surgery. Moreover, patients with a spherical refractor error over 4.00 dioptres or a cylindrical refractor error over 2.00 dioptres were excluded to restrict the influence of axial length on proper OCTA assessment.

All RA patients underwent a comprehensive ophthalmological examination, including assessment of best-corrected visual acuity (BCVA) on standard ETDRS LogMAR charts at $4 \mathrm{~m}$, slit lamp examination, tonometry, dilated fundus examination, structural and angiographic optical coherence tomography (OCT/OCTA) evaluation, and static visual field examination, at the baseline and after a one-year follow-up.

OCTA technology enables the retinal and subretinal vascular networks to be reconstructed by encoding the source signal derived from the movement of erythrocytes inside the vessels; the trajectory of the vascular flows thus makes it possible to create a map of the vascular network. The retinal microvascular network imaging was analyzed by OCTA (Optovue XR Avanti, Fremont, CA, USA) and performed on all patients. The scans were centered on the fovea and the images acquired with the $6 \times 6 \mathrm{~mm}$ protocol. After acquisition, the internal software automatically delineates the superficial capillary plexus (SCP) and deep capillary plexus (DCP). The AngioVue software detects the SCP as the layer included between approximately $3 \mu \mathrm{m}$ below the internal limiting membrane and $15 \mu \mathrm{m}$ below the inner plexiform layer, while the DCP lies 15 to $70 \mu \mathrm{m}$ below the inner plexiform layer. SCP and DCP vessel density (VD), central foveal thickness (CFT) and foveal avascular zone (FAZ) values were collected. In more detail, the VD of the global area, foveal area (circle $1 \mathrm{~mm}$ in diameter centered on the fovea), and parafoveal region (between two concentric circles of $1 \mathrm{~mm}$ diameter and $3 \mathrm{~mm}$ diameter) of the SCP and DCP were calculated, providing specific values for the temporal, nasal, superior and inferior regions of the parafoveal area. Poor quality OCTA scans (signal strength below index 50, motion artefacts, and inappropriate segmentation) were excluded from the analysis. Structural analysis was performed at the baseline examination and at the one-year follow-up to estab- 
lish the normal retinal architecture and if necessary identify anatomical changes related to HCQ toxicity, especially outer nuclear layer damage. Each scan was evaluated by two independent retinal specialists; uncertain cases were collectively discussed and categorized.

Static visual field examination was performed by automated visual field testing, using a Humphrey computerized visual field device (Carl Zeiss Meditec Inc., Dublin, CA, USA), after applying refractive correction appropriate for the test distance. Central visual field testing was done using a 10-2 threshold central test program, which scans a 10 degree area at intervals of 2 degrees. At the baseline examination, a visual field with more than $33 \%$ false positive or false negative responses or loss of fixation was considered unreliable and the patient was invited to repeat the test and improve the execution.

At the conclusion of the one-year follow-up, a loss of more than $5 \mathrm{~dB}$ at three or more adjacent points or over $10 \mathrm{~dB}$ at a single point was taken as evidence of visual field deterioration. Consequently, the presence of visual field damage marked the case as displaying HCQ toxicity.

HCQ was administered following the dosage recommendation of a maximum daily HCQ use of $\leq 5.0 \mathrm{mg} / \mathrm{kg}$ of actual body weight per day [27]. In addition, patients weighing more than $80 \mathrm{~kg}$ received no more than $400 \mathrm{mg}$ HCQ per day.

The primary outcome measure was the assessment of the VD alterations in the retinal capillary plexus in subjects affected by recently diagnosed and treatment-naïve RA, and the comparison of the data at the one-year follow-up examination.

The Shapiro-Wilk test was used to check the assumption of normality of the variables. Parametric (t-test) and non-parametric (Mann-Whitney) tests were applied according to variable distribution. Demographic and clinical data were summarized for descriptive statistic purposes, presenting the mean value as well as the standard deviation. All statistical comparisons were performed using 2-tailed significance tests, with $p<0.05$ considered statistically significant. MedCalc Statistical Software (version 19.8, Ostend, Belgium) was used for all analyses.

\section{Results}

Sixteen treatment-naïve RA patients satisfied inclusion and exclusion criteria, completed the one-year follow-up study and were considered fully eligible for the study's purposes. The patients' mean age was $61.2 \pm 12$ years and the female:male ratio was8:8 (Table 1).

Table 1. Demographic and clinical parameters of rheumatoid arthritis (RA) patients.

\begin{tabular}{cc}
\hline Parameters & Mean/Ratio \\
\hline Number of patients & 16 \\
Age (mean \pm SD, years) & $61.2 \pm 12$ \\
Female:male ratio & $8: 8$ \\
Symptoms duration related to RA (Mean \pm SD, months) & $5.6 \pm 2.7$ \\
Previous or associated DMARDs (disease-modifying & None \\
antirheumatic drugs),including ciclosporin, cyclophosphamide, & \\
leflunomide, methotrexate, mycophenolate, sulfasalazine. & \\
DAS28 (disease activity score) & $4.80 \pm 0.5$ \\
Baseline (mean \pm SD) & $3.07 \pm 0.4$ \\
Final examination (mean \pm SD) & $85 \pm 12$ \\
Body weight (Kg, mean \pm SD) & $400 \mathrm{mg}$ \\
HCQ daily dose & $146 \mathrm{~g}$ \\
HCQ cumulative dose at the one-year examination & $1.74 \pm 0.22 \mathrm{~g}$ \\
\hline HCQ cumulative dose $/$ Kg of actual body weight at the one-year & \\
examination & \\
\hline
\end{tabular}


Mean symptom duration related to RA was $5.6 \pm 2.7$ months and all patients obtained pain relief within four to six months after starting therapy. No patient discontinued therapy during the study period or changed the treatment's starting dose. Moreover, no additional drug was administered in association with HCQ. All patients received $400 \mathrm{mg}$ $\mathrm{HCQ}$ a day, divided into two doses, $12 \mathrm{~h}$ apart, giving a cumulative dose of $146 \mathrm{~g}$ at the one-year examination.

BCVA was $84 \pm 1.8$ letter score (20/20-, Snellen) at the baseline examination and remained stable at one year examination. Visual field testing was normal at the baseline and final examinations in all patients.

Structural OCT analysis did not reveal any alterations in the retinal architecture related to HCQ toxicity, with the two retinal specialists in total agreement.

Stable visual acuity, absence of abnormalities on slit-lamp fundus examination, normal VF assessment and structural OCT analysis meant all patients could be regarded as normal and without HCQ toxicity at the end of one-year follow-up.

Signal strength index in the RA group was $76 \pm 7$ and $77 \pm 4$ at the baseline and 12-month follow-up examinations, respectively. All OCTA scans were considered eligible for the baseline and final evaluations.

OCTA data analysis showed no statistically significant change in the VD in all parameters at the one-year follow-up, both in the SCP and in the DCP (Table 2), compared with the baseline value. No significant differences in FAZ values were registered between the baseline and the one-year follow-up.

Table 2. Vessel density analysis of superficial capillary plexus (SCP), deep capillary plexus (DCP) and foveal avascular zone (FAZ) values.

\begin{tabular}{cccc}
\hline & $\begin{array}{c}\text { Baseline } \\
\text { (Mean } \pm \text { SD) }\end{array}$ & $\begin{array}{c}\text { 12-Month } \\
\text { Examination } \\
\text { (Mean } \pm \text { SD) }\end{array}$ & $p$ Value \\
\hline Vessel Density (\%) in RA Patients & & & \\
\hline SCP Global Area & $45.3 \pm 4.6$ & $45.7 \pm 3.8$ & 0.78 \\
SCP CSF & $25.2 \pm 7.5$ & $23.7 \pm 5.1$ & 0.46 \\
SCP Inner Ring & $47.8 \pm 6.3$ & $47.4 \pm 4.9$ & 0.85 \\
SCP Temporal & $47.9 \pm 6.9$ & $47.0 \pm 5.4$ & 0.69 \\
SCP Superior & $47.2 \pm 6.1$ & $48.3 \pm 5.2$ & 0.59 \\
SCP Nasal & $46.9 \pm 6.8$ & $46.6 \pm 4.9$ & 0.86 \\
SCP Inferior & $49.1 \pm 6.7$ & $47.8 \pm 5.2$ & 0.50 \\
DCP Global Area & $45.7 \pm 6.3$ & $46.3 \pm 6.2$ & 0.77 \\
DCP CSF & $37.3 \pm 8.9$ & $40.3 \pm 5.5$ & 0.11 \\
DCP Inner Ring & $51.2 \pm 5.0$ & $52.06 \pm 4.9$ & 0.60 \\
DCP Temporal & $52.1 \pm 5.7$ & $53.7 \pm 5.3$ & 0.38 \\
DCP Superior & $50.4 \pm 5.9$ & $52.2 \pm 5.6$ & 0.25 \\
DCP Nasal & $51.7 \pm 6.0$ & $52.3 \pm 5.2$ & 0.68 \\
DCP Inferior & $50.8 \pm 4.5$ & $49.9 \pm 5.12$ & 0.34 \\
FAZ Value mm ${ }^{2}$ & $0.22 \pm 0.07$ & $0.22 \pm 0.06$ & 0.95 \\
\hline
\end{tabular}

Abbreviations: RA—rheumatoid arthritis; SCP—superficial capillary plexus; DCP—deep capillary plexus; FAZ— foveal avascular zone; CSF—central subfield. 
A representative case at the baseline and one-year follow-up is illustrated in Figure 1.

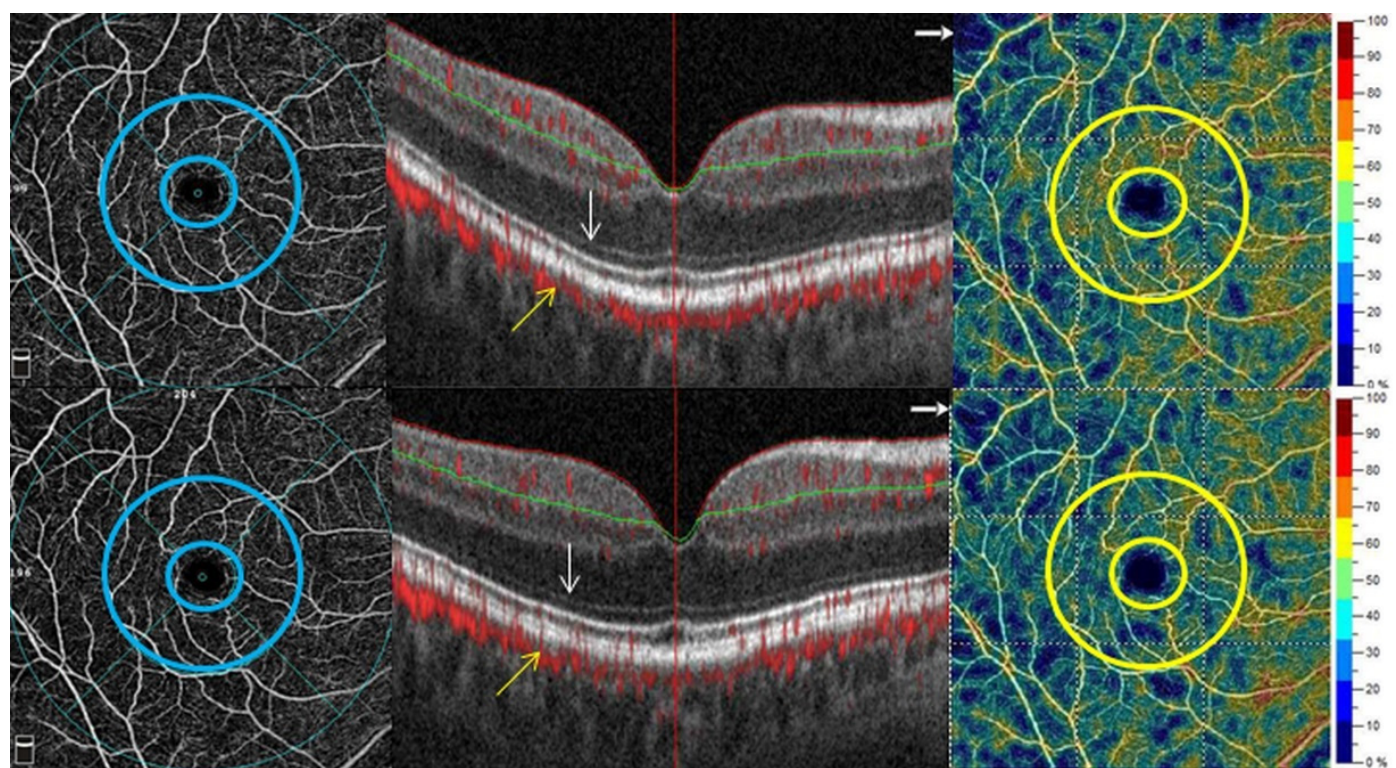

Figure 1. Optical coherence tomography angiography (OCTA) and capillary perfusion density analysis. Legend: Top row: Left, example of en face OCTA of rheumatoid arthritis patient. The foveal area is identified by a circle $1 \mathrm{~mm}$ in diameter centered on the fovea, the parafoveal region is included between the two concentric circles of $1 \mathrm{~mm}$ diameter and $3 \mathrm{~mm}$ diameter; center, structural optical coherence tomography, showing the complete integrity of the layers and flow signals; right, full retina capillary vessel density analysis. Bottom row: No changes in the retinal architecture and vessel density at one-year follow-up can be seen, compared with baseline images; in particular, the integrity of the retinal inner and outer segments (white arrow) and retinal pigment epithelium (yellow arrow) are well represented; in parallel, no significant change in the vessel density may be observed within 1 and $3 \mathrm{~mm}$ circles.

\section{Discussion}

A percentage varying from $1.6 \%$ to $20 \%$ of subjects undergoing therapy with chloroquine/HCQ for autoimmune diseases, including rheumatoid arthritis, manifest retinal toxicity. This is characterized by damage to the outer retinal layers, associated with reduced visual acuity and visual sensitivity, mainly experienced in the central 10 degrees of the visual field [27-29]. Direct toxicity to EPR seems to be the most commonly cited pathogenetic mechanism responsible for the final loss of retinal integrity and visual function. More recently, some authors have advanced the hypothesis that the retinal capillary circulation might be involved in the pathogenesis of retinal toxicity. In particular, it has been suggested that chloroquine/HCQ could reduce the VD of the macular retinal capillary network and subsequently lead to a reduction of blood flow. The reduction of the VD of the retinal capillary plexus has been demonstrated by OCTA in subjects with RA undergoing chronic therapy for five to 10 years, in comparison with a healthy population [14]. A greater reduction of VD was detected in the subgroup of patients with early retinal toxicity [16].

However, these assumptions were made in the absence of a clear understanding of the anatomical characteristics of the retinal capillary plexus in subjects with RA. In a previous study, we demonstrated that a reduction in the VD of SCP can be detected early in naïve patients with recent onset of symptoms related to RA, compared to a group of healthy controls [25]. This finding may be complementary with the alterations in the retinal macro-circulation already highlighted by Anyfanti et al [24]. In a population study of 113 RA patients, the authors demonstrated a reduction in the caliber of the arterial vessels and a reduction in the arteriovenous ratio, in comparison with a control group.

A vascular remodeling of the retinal micro- and macro-circulation, thus, seems to be a characteristic of RA. Primary alterations of the circulation in the large vessels and especially in the peripheral microvessels in patients with RA, including alterations of peripheral 
capillary circulation of the extremities of the limbs and in the oral and labial mucosa, have been previously described and imply the presence of complex pathogenic mechanisms involving cell-mediated inflammation, immune complex-mediated inflammation and autoantibody-mediated inflammation, in addition to endothelial cell activation, and elevated levels of endothelin-1, potentially inducing vascular dysregulation [17-24,30-34].

The current study produces evidence of the stability of the VD in the SCP and DCP in patients with RA receiving HCQ over a one-year follow-up. In this cohort of patients, no retinal toxicity was demonstrated on structural OCT and visual field examination.

Drugs might need to be administered for a longer period in order to reduce the retinal VD associated with HCQ therapy. Furthermore, the progressive reduction in VD might be affected by the cumulative dose rather than the daily dose.

Only a long, monitored follow-up study can help clarify whether there is a real progressive reduction in VD relative to HCQ dose, cumulative dosage or time of administration. However, any data should be appraised in comparison with a group of RA subjects not undergoing HCQ treatment in order to pinpoint whether the evolution of the VD is spontaneous or is part of a specific pattern of VD changes related to the development of RA. It is equally important to determine the progression of the condition and vascular patterns in subjects presenting HCQ retinal toxicity, in order to identify potential biomarkers indicating subjects at high risk of retinal toxicity.

Previous studies analyzing VD reduction during HCQ therapy share a number of limitations. In their investigations, Bulut and Goker provided clear evidence of a reduction of VD in patients at high risk of HCQ-induced retinal toxicity (HCQ therapy > five years) $[11,15]$. Bulut's study observed a significant reduction of VD in the high-risk group compared with the low-risk group (HCQ therapy < five years); similarly, Goker's research followed a group of patients receiving HCQ therapy over a period of five years in comparison with a control group, finding a significant reduction of VD in the former. However, neither study categorized the patients according to subtypes of autoimmune diseases, so that data regarding LES, RA and other disorders were pooled. LES is especially important in this context, as it is characterized by primary retinal vascular changes acting as markers of a vaso-occlusive retinopathy, including cotton-wool spot, hard exudates, arteriolar attenuation, vasculitis and arterial occlusion [35]. Moreover, LES-related retinal ischemia may be confused with macula damage due to HCQ [13]. OCTA studies should therefore take into account retinal vascular changes related to the different autoimmune diseases when considering changes involving the VD.

The study carried out by Tarakcioglu and colleagues also fails to include a subclassification of autoimmune diseases, thus compromising a proper evaluation of retinal VD [36]. The authors examined VD and retinal thickness in a cohort of 102 eyes of 102 patients with autoimmune disease, comparing data of 70 patients included in the treatment group with 32 naïve patients in the control group. The vascular density values in the control group were matched by those in the treatment group $(p>0.05)$, supporting the conclusion that HCQ administered over a mean time of 73 months may not alter the retinal VD. Unfortunately, the data were not categorized by autoimmune disease, and low-risk (HCQ therapy < five years) and high-risk (HCQ therapy > five years) patients were not compared separately with the control group's naïve patients.

The current study has some clear limitations, the most obvious being the small sample size. However, this is the first study to include naïve RA patients and those NB receiving HCQ as first line therapy, monitored with visual field and structural OCT and OCTA in a prospective investigation. Our protocol identifies another limitation, prompted by force of circumstance, namely the absence of multifocal electroretinogram examination (MF-ER) and fundus autofluorescence (FAF) among the inclusion criteria for the early detection of HCQ retinopathy, as recommended by the American Academy of Ophthalmology. Since the current study was carried out during the COVID-19 pandemic, we decided to restrict examinations to those that were quickest and most informative, so as to avoid prolonged stays in the hospital. This ruled out the MF-ERG and FAF. MF-ERG and FAF examinations 
could identify specific, early abnormalities and thus categorize some eyes differently, suggesting possible correlations between functional impairment and retinal VD changes. Finally, bearing in mind that HCQ's side effects are usually obvious after five years or more of use, only a long-term follow-up study can confirm the vascular remodeling in RA patients undergoing HCQ therapy and the associated patterns of vascular changes. An optimal model would include a control group of RA patients not undergoing HCQ therapy. This would offer the chance to analyze the effects of HCQ therapy on retinal capillary plexuses in greater depth, as well as providing the opportunity to examine the changes in VD during the natural evolution of the disease and confirm the early alterations of the retinal SCP previously demonstrated [25]. Forming such a control group would give rise to ethical questions regarding the decision not to treat RA patients for a long period or to opt for alternative therapeutic options, whose effects on the retinal capillary network are not yet fully understood.

\section{Conclusions}

In conclusion, our study demonstrates retinal VD in the SCP and DCP is stable in previously naïve RA patients during one year of HCQ therapy. The significant decrease of VD in the SCP revealed at the baseline examination, compared with an age- and sexmatched control group in our preliminary study [25], seems not to be affected by HCQ therapy at least in short-term follow-up.

Author Contributions: Conceptualization, P.I.; methodology, P.I.; validation, P.I., S.D.P., A.B., M.V. and M.P.; formal analysis.; investigation, P.I., S.D.P., A.B., M.V. and M.P.; resources, P.I.; data curation, P.I. and S.D.P.; writing—original draft preparation, P.I.; writing—review and editing, P.I., S.D.P., A.B., M.V. and M.P. All authors have read and agreed to the published version of the manuscript.

Funding: The authors have not declared a specific grant for this research from any funding agency in the public, commercial or not-for-profit sectors.

Institutional Review Board Statement: The study fully respected the tenets of the Declaration of Helsinki and the institutional review board approval of G.B. Bietti was obtained before starting the study. The IRB approved the observational study without providing a specific ID. In general, noninterventional studies are communicated to the scientific committee which takes current researches into account and deposits research proposals.

Informed Consent Statement: Each patient was carefully informed about the purpose of the research and provided signed consent to all procedures and data analysis.

Data Availability Statement: The data that support the findings of this study are available from the corresponding author, (P.I.), upon reasonable request.

Acknowledgments: The research for this paper was financially supported by Italian Ministry of Health and Fondazione Roma.

Conflicts of Interest: The authors declare no conflict of interest. The funders had no role in study design, data collection and analysis, decision to publish, or preparation of the manuscript.

\section{References}

1. Ben-Zvi, I.; Kivity, S.; Langevitz, P.; Shoenfeld, Y. Hydroxychloroquine: From Malaria to Autoimmunity. Clin. Rev. Allergy Immunol. 2012, 42, 145-153. [CrossRef]

2. Mian, A.; Ibrahim, F.; Scott, D.L. A systematic review of guidelines for managing rheumatoid arthritis. BMC Rheumatol. 2019, 3, 1-13. [CrossRef]

3. Daien, C.; Hua, C.; Gaujoux-Viala, C.; Cantagrel, A.; Dubremetz, M.; Dougados, M.; Fautrel, B.; Mariette, X.; Nayral, N.; Richez, C.; et al. Update of French society for rheumatology recommendations for managing rheu-matoid arthritis. Jt. Bone Spine 2019, 86, 135-150. [CrossRef]

4. Fava, A.; Petri, M. Systemic lupus erythematosus: Diagnosis and clinical management. J. Autoimmun. 2019, 96, 1-13. [CrossRef] [PubMed]

5. Bowman, S.J. Primary Sjögren's syndrome. Lupus 2018, 27 (Suppl. 1), 32-35. [CrossRef] [PubMed]

6. Katzung, B.G.; Masters, S.B.; Trevor, A.J. Basic and Clinical Pharmacology, 12th ed.; McGraw-Hill Companies: New York, NY, USA, 2011; Chapter 36; p. 644. 
7. Yusuf, I.H.; Sharma, S.; Luqmani, R.; Downes, S.M. Hydroxychloroquine retinopathy. Eye (London) 2017, 31, 828-845. [CrossRef]

8. Constable, S.; Pirmohamed, M. Drugs and the retina. Expert Opin. Drug Saf. 2004, 3, 249-259. [CrossRef] [PubMed]

9. Schrezenmeier, E.; Dörner, T. Mechanisms of action of hydroxychloroquine and chloroquine: Implications for rheumatology. Nat. Rev. Rheumatol. 2020, 16, 155-166. [CrossRef] [PubMed]

10. Abdulaziz, N.; Shah, A.R.; McCune, W.J. Hydroxychloroquine: Balancing the need to maintain therapeutic levels with ocular safety: An update. Curr. Opin. Rheumatol. 2018, 30, 249-255. [CrossRef] [PubMed]

11. Bulut, M.; Akıdan, M.; Gözkaya, O.; Erol, M.K.; Cengiz, A.; Çay, H.F. Optical coherence tomography angiography for screening of hydroxychloro-quine-induced retinal alterations. Graefe's Arch. Clin. Exp. Ophthalmol. 2018, 256, 2075-2081. [CrossRef]

12. Conigliaro, P.; Cesareo, M.; Chimenti, M.S.; Triggianese, P.; Canofari, C.; Aloe, G.; Nucci, C.; Perricone, R. Evaluation of retinal microvascular density in patients affected by systemic lu-pus erythematosus: An optical coherence tomography angiography study. Ann. Rheum. Dis. 2019, 78, 287-289. [CrossRef] [PubMed]

13. Forte, R.; Haulani, H.; Dyrda, A.; Jürgens, I. Swept source optical coherence tomography angiography in patients treated with hydroxychloroquine: Correlation with morphological and functional tests. Br. J. Ophthalmol. 2019. [CrossRef] [PubMed]

14. Ozek, D.; Onen, M.; Karaca, E.E.; Omma, A.; Kemer, O.E.; Coşkun, Ç. The optical coherence tomography angiography findings of rheumatoid arthritis patients taking hydroxychloroquine. Eur. J. Ophthalmol. 2018, 29, 532-537. [CrossRef] [PubMed]

15. Goker, Y.S.; Atılgan, C.U.; Tekin, K.; Kızıltoprak, H.; Yetkin, E.; Karahan, N.Y.; Koc, M.; Kosekahya, P. The Validity of Optical Coherence Tomography Angiography as a Screening Test for the Early Detection of Retinal Changes in Patients with Hydroxychloroquine Therapy. Curr. Eye Res. 2018, 44, 311-315. [CrossRef]

16. Akhlaghi, M.; Kianersi, F.; Radmehr, H.; Dehghani, A.; Naderi Beni, A.; Noorshargh, P. Evaluation of optical coherence tomography angiography parameters in patients treated with Hydroxychloroquine. BMC Ophthalmol. 2021, 21, 209. [CrossRef] [PubMed]

17. Scardina, G.A.; Messina, P. Microvascular abnormalities in patients with rheumatoid arthritis. Ann. Anat.-Anat. Anz. 2006, 188, 425-429. [CrossRef] [PubMed]

18. Radić, M.; Kaliterna, D.M.; Radić, J. Overview of vasculitis and vasculopathy in rheumatoid arthritis-Something to think about. Clin. Rheumatol. 2013, 32, 937-942. [CrossRef]

19. Makol, A.; Matteson, E.L.; Warrington, K.J. Rheumatoid vasculitis: An update. Curr. Opin. Rheumatol. 2015, 27, 63-70. [CrossRef]

20. Guillevin, L.; Dörner, T. Vasculitis: Mechanisms involved and clinical manifestations. Arthritis Res. Ther. 2007, 9, S9. [CrossRef]

21. Tanasescu, C.; Jurcut, C.; Jurcut, R.; Ginghina, C. Vascular disease in rheumatoid arthritis: From subclinical lesions to cardiovascular risk. Eur. J. Intern. Med. 2009, 20, 348-354. [CrossRef]

22. Pieringer, H.; Pichler, M. Cardiovascular morbidity and mortality in patients with rheumatoid arthritis: Vascular altera-tions and possible clinical implications. QJM Int. J. Med. 2011, 104, 13-26. [CrossRef] [PubMed]

23. Sandoo, A.; Veldhuijzen van Zanten, J.J.; Metsios, G.S.; Carroll, D.; Kitas, G.D. Vascular function and morphology in rheuma-toid arthritis: A systematic review. Rheumatology (Oxford) 2011, 50, 2125-2139. [CrossRef] [PubMed]

24. Anyfanti, P.; Triantafyllou, A.; Gkaliagkousi, E.; Koletsos, N.; Athanasopoulos, G.; Zabulis, X.; Galanopoulou, V.; Aslanidis, S.; Douma, S. Retinal vessel morphology in rheumatoid arthritis: Association with systemic inflammation, subclinical atherosclerosis, and cardiovascular risk. Microcirculation 2017, 24, e12417. [CrossRef]

25. Iacono, P.; Da Pozzo, S.; Bedendo, A.; Arrigo, A.; Parravano, M.; Varano, M.; Parodi, M.B. OCT retinal angiography features in patients with rheumatoid arthritis: A pilot study. Eur. J. Ophthalmol. 2021. [CrossRef]

26. Aletaha, D.; Neogi, T.; Silman, A.J.; Funovits, J.; Felson, D.T.; Bingham, C.O., III; Birnbaum, N.S.; Burmester, G.R.; Bykerk, V.P.; Cohen, M.D.; et al. 2010 Rheumatoid arthritis classification criteria: An American College of Rheumatolo-gy/European League Against Rheumatism collaborative initiative. Arthritis Rheum. 2010, 62, 2569-2581. [CrossRef]

27. Melles, R.B.; Marmor, M.F. The Risk of Toxic Retinopathy in Patients on Long-term Hydroxychloroquine Therapy. JAMA Ophthalmol. 2014, 132, 1453. [CrossRef]

28. Marshall, E.; Robertson, M.; Kam, S.; Penwarden, A.; Riga, P.; Davies, N. Prevalence of hydroxychloroquine retinopathy using 2018 Royal College of Ophthalmologists diagnostic criteria. Eye (London) 2021, 35, 343-348. [CrossRef]

29. Eo, D.R.; Lee, M.G.; Ham, D.I.; Kang, S.W.; Lee, J.; Cha, H.S.; Koh, E.; Kim, S.J. Frequency and Clinical Characteristics of Hydroxychlo-roquine Retinopathy in Korean Patients with Rheumatologic Diseases. J. Korean Med. Sci. 2017, 32, 522-527. [CrossRef]

30. Scott, D.G.; Bacon, P.A.; Allen, C.; Elson, C.J.; Wallington, T. IgG rheumatoid factor, complement and immune complexes in rheumatoid synovitis and vasculitis: Comparative and serial studies during cytotoxic therapy. Clin. Exp. Immunol. 1981, 43, 54-63. [PubMed]

31. Turesson, C.; Jacobsson, L.T.H.; Sturfelt, G.; Matteson, E.L.; Mathsson, L.; Ronnelid, J. Rheumatoid factor and antibodies to cyclic citrullinated peptides are associated with severe extra-articular manifestations in rheumatoid arthritis. Ann. Rheum. Dis. 2006, 66, 59-64. [CrossRef] [PubMed]

32. Anyfanti, P.; Gkaliagkousi, E.; Triantafyllou, A.; Zabulis, X.; Dolgyras, P.; Galanopoulou, V.; Aslanidis, S.; Douma, S. Dermal capillary rarefaction as a marker of microvascular damage in patients with rheumatoid arthritis: Association with inflammation and disorders of the macrocirculation. Microcirculation 2018, 25, e12451. [CrossRef]

33. Rajaei, A.; Dehghan, P.; Amiri, A. Nailfold Capillaroscopy in 430 patients with Rheumatoid Arthritis. Casp. J. Intern. Med. 2017, 8 , 269-274. [CrossRef] 
34. Klimek, E.; Sulicka, J.; Gryglewska, B.; Skalska, A.; Kwaśny-Krochin, B.; Korkosz, M.; Grodzicki, T.K. Alterations in skin microvascular function in patients with rheumatoid arthritis and ankylosing spondylitis. Clin. Hemorheol. Microcirc. 2017, 65, 77-91. [CrossRef] [PubMed]

35. Kharel, R.; Shah, D.N.; Singh, D.; Sitaula, R.K. Role of lupus retinopathy in systemic lupus erythematosus. J. Ophthalmic Inflamm. Infect. 2016, 6, 15. [CrossRef] [PubMed]

36. Tarakcioglu, H.N.; Ozkaya, A.; Yigit, U. Is optical coherence tomography angiography a useful tool in the screening of hydroxychloroquine retinopathy? Int. Ophthalmol. 2021, 41, 27-33. [CrossRef] [PubMed] 